\title{
Correlations Between Severity of Coronary Calcification and Impairment of Left Ventricular Ejection Fraction
}

\author{
Suciu Zsuzsanna1, Benedek Theodora¹, Jakó Beáta², Benedek I1 \\ ${ }^{1}$ Clinic of Cardiology, Discipline of Internal Medicine 6, University of Medicine and Pharmacy, Tîrgu Mureș, Romania \\ 2 Clinic of Cardiology, County Emergency Clinical Hospital, Tîrgu Mureș, Romania
}

\begin{abstract}
Introduction: 64 multislice CT angiography is a recently introduced imaging technique, increasingly being used as a tool to show the coronary arteries in three-dimensional visualization. One of the advantages of this method is the ability to estimate the degree of calcification of atheromatous plaques via coronary calcium score calculation, which correlates with the severity score of ateromatous systemic burden. The aim of this study was to evaluate the relationship between the severity of coronary calcification, expressed by calcium score, and the left ventricular ejection fraction (LVEF).

Material and methods: This retrospective study included 81 patients with symptoms of angina and ECG modifications (at rest or during exercise). Echocardiography and 64 multislice CT angiography were performed in all patients to assess the LVEF and Ca scoring.

Results: Calcium score was lower than 100 in 62 patients (50.22\%), between 100 and 400 in 11 patients (8.91\%), and higher than 400 in 8 patients (6.48\%). Mean LVEF was 53.52\%, 17 patients having an LVEF of less than $50 \%$. In patients with calcium score less than 100 , the corresponding ejection fraction was normal: $55.29 \%$, while in coronary arteries with extensive calcifications (calcium score > 400), the LVEF was significantly lower, $50.5 \%(p=0.004)$.

Conclusions: High Calcium score is positively correlated with LVEF reduction, and a high value for calcium score indicates an increased probability of reduced left ventricular ejection fraction.
\end{abstract}

Keywords: multislice 64 computed tomography, coronary calcium score, left ventricular ejection fraction

Received: 19 July 2013

\section{Introduction}

Multislice 64 CT angiography is a recently introduced imaging technique, being used increasingly as a tool to show the coronary arteries in three-dimensional visualization. The method provides high definition images based on 2D and 3D image reconstructions, extremely useful for assessment of coronary artery disease, similar with those obtained by coronary angiography, but in a noninvasive way, in the same time allowing identification and characterization of both calcified and non-calcified coronary atherosclerotic plaques, with high sensitivity and specificity $[1,2]$.

One of the advantages of this method is the ability to estimate the degree of calcification of atheromatous plaques via coronary calcium score calculation, which correlates with the severity score of atheromatous systemic burden. Calcium scoring is a newly introduced application of Cardiac CT, serving as an indirect marker of coronary artery atherosclerotic load. The coronary artery calcium score, usually calculated using the Agatston method and expressed in Agatston units, represents a summation of all calcification accumulated in the lesions of the coronary arteries and has been demonstrated in several trials to represent a strong predictor of future cardiovascular events.

Correspondence to: Theodora Benedek

E-mail: hintea_teodora@yahoo.com
A high value of coronary calcium score has a powerful diagnostic value even before the first symptoms or signs of cardiac ischemia develop. It has been shown that coronary calcium is detectable in the vast majority of patients with acute coronary syndromes, and the amount of calcium in these patients is higher than in non-ACS population $[3,4]$.

According to standard classifications, the patient's calcium score is correlated with the probability of coronary artery disease (Table I). From a clinical perspective, current guidelines suggest that a low (or zero) score is associated with a low prevalence of coronary artery ischemia and low cardiac event rates over the next 5 years, while higher calcium scores are associated with higher rates of events [5].

It is well-known that a significant obstructive coronary artery disease is in many cases associated with a decrease in left ventricular function.

The aim of this study was to evaluate the relationship between the severity of coronary calcification, expressed by calcium score, and the left ventricular ejection fraction (LVEF).

\section{Material and methods}

This retrospective study included 81 patients with symptoms of angina and ECG modifications (at rest or during exercise). Echocardiography and 64 multislice CT angiog- 
Table I. Interpretation of calcium score

\begin{tabular}{|c|c|c|}
\hline Calcium Score & Presence of Plaque & \\
\hline 0 & $\begin{array}{l}\text { No evidence of } \\
\text { plaque }\end{array}$ & $\begin{array}{l}\text { A calcium score of } 0 \text { indicates } \\
\text { absence of detected calcium and an } \\
\text { extremely low likelihood }(<1 \%) \text { of any } \\
\text { coronary artery disease }\end{array}$ \\
\hline $1-100$ & $\begin{array}{l}\text { Mild evidence of } \\
\text { plaque }\end{array}$ & $\begin{array}{l}\text { Low probability of coronary artery } \\
\text { disease }\end{array}$ \\
\hline $100-400$ & $\begin{array}{l}\text { Moderate evidence } \\
\text { of plaque }\end{array}$ & $\begin{array}{l}\text { Non-obstructive coronary heart } \\
\text { disease likely, although obstructive } \\
\text { disease possible }\end{array}$ \\
\hline Over 400 & $\begin{array}{l}\text { Extensive evidence } \\
\text { of plaque }\end{array}$ & $\begin{array}{l}\text { High likelihood of at least one signifi- } \\
\text { cant coronary stenosis }\end{array}$ \\
\hline
\end{tabular}

raphy were performed in all patients to assess the LVEF and Ca scoring.

The two-dimensional echocardiography was performed using the equipment Siemens-Acuson X 300. Standard Mmode, 2D images and Doppler and colour-Doppler data were acquired from the parasternal and apical views (4-, 2-, and 3-chamber). Left Ventricular diameters, and enddiastolic thickness of the interventricular septum and the posterior wall were measured on the parasternal long-axis M-mode recordings. Left Ventricular end-systolic and enddiastolic volumes were assessed and LVEF was calculated from the apical 4- and 2-chamber views using the Simpson's rule, and expressed as a percentage. An LV ejection fraction higher than $55 \%$ was considered normal, an LV ejection fraction between 50 and $55 \%$ was considered intermediary, while an LV ejection fraction lower than 50\% was considered reduced.

In all cases electrocardiographically (ECG) - gated multislice cardiac CT was performed using the equipment Somatom Sensation multislice 64 (Siemens) with the following scanning parameters: tube current $150 \mathrm{mAs}$, retrospective gating ECG synchronization, estimated radiation exposure $1.7 \pm 0.8 \mathrm{mSv}$. The extent of calcium in the coronary arteries was assessed during the precontrast scans taken at the start of imaging. Results were obtained rapidly - the CT scan takes about 20 seconds. However, extra time, approximately 5 minutes was needed to evaluate the results. We classified the coronary calcium scores into 3 categories
$(<100,100-400$, and $>400)$. The following parameters were calculated by the Syngo CT software, based on separation and modification of lesions within a defined volume (depth in $\mathrm{mm}$ ) or on 2D-slices: area (in $\mathrm{mm}^{2}$ ), peak density (in Hounsfield Units), volume (in $\mathrm{mm}^{3}$ ), calcium mass ( $\mathrm{mg}$ Calcium Hydroxyapatite), and score (Agatston method).

The calcium scoring was calculated, which represents the content of calcium in the 3 coronary arteries (RCA, LAD, CX) and left main trunk, obtained by addition of calcium content in all the segments of the coronary tree (Figure 1).

Statistical analysis was performed using Graph InStat Pad softwares. Continuous values are expressed as the mean and standard deviation, and statistical significance was determined using the Mann-Whitney test. Statistical significance was considered for a $\mathrm{p}$ value $<0.05$, and all $\mathrm{p}$ values were 2 -sided.

\section{Results}

The study group consisted of 81 patients ( 35 men and 46 women; the average age was 59.6 years) who were referred for two-dimensional echocardiography and 64 multislice CT angiography in the same day because of clinically suspected coronary artery disease.

Calcium score was lower than 100 in 62 patients (50.22\%), between 100 and 400 in 11 patients (8.91\%), and higher than 400 in 8 patients (6.48\%) (Figure 2). Regarding the echocardiographic characteristics, the mean LVEF was $53.52 \%, 17$ patients having an LVEF of less than $50 \%$.

In patients with calcium score less than 100, the corresponding ejection fraction was normal: 55.29\%, while in coronary arteries with extensive calcifications (calcium score $>400$ ), the LVEF was significantly lower, $50.5 \%$ ( $\mathrm{p}=$ 0.004) (Figure 3).

\section{Discussion}

The most recent generations of multislice CT with the ability to acquire 64 slices simultaneously allow relatively robust morphological and functional imaging of the heart, enabling quantification of coronary artery stenosis and plaque
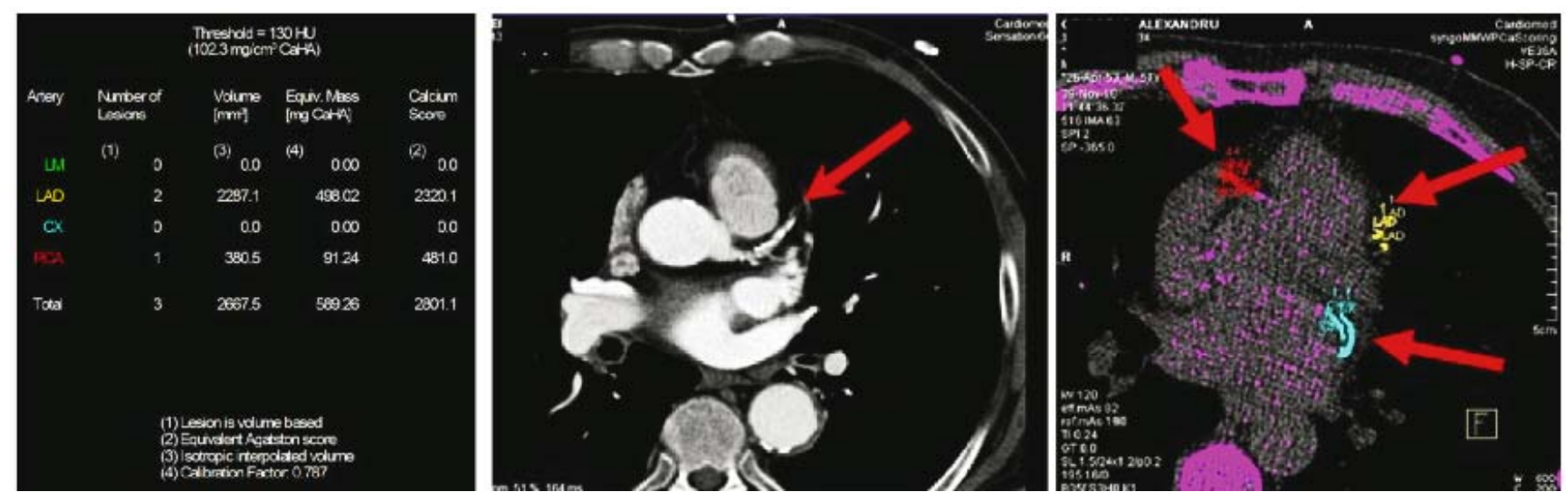

Fig. 1. Analysis of coronary artery calcification and presence of calcium in the coronary arteries 


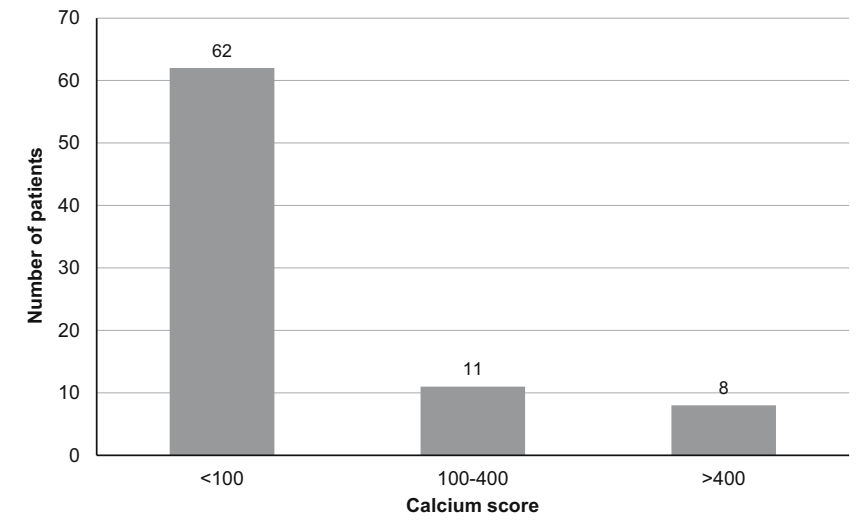

Fig. 2. Calcium score dispersion in patient population

burden, thus helping us to appreciate the indication for revascularization and select the proper method for it $[6,7]$.

Computed tomography calcium scoring is a safe, rapid and new non-invasive method and screening tool for detecting calcium in the coronary arteries. The Agatston Score usually serves for CT quantification of the exact amount of coronary calcium accumulation, on the basis of the area and the CT density of calcified lesions [8]. Clinically, coronary calcium scoring can be used as a predictor of future cardiovascular events, as demonstrated in several international trials, independently of standard risk factors, and enhance Framingham risk stratification categories. The American Heart Association has given the Coronary Artery Calcium Scoring a level II recommendation for patients who have an intermediate risk for heart disease. It can be used for a more accurate classification of patients according to their coronary risk in order to establish the most appropriate management for each case $[9,10]$.

However, our study indicates that there is a strong correlation between increase in coronary calcium score and decrease of Left ventricular function in patients with coronary artery disease. We found a significantly lower left ventricular ejection fraction in patients with a coronary calcium score higher than 400 as compared with the ejection fraction of patients with coronary calcium score less than 100 , two standard thresholds for defining the severity of calcium accumulation within the coronary arteries.

We showed that coronary calcium score calculated with multislice computed tomography is increased in patients with low ejection fraction, representing a marker of high cardiovascular risk.

Therefore the coronary calcium score, easily to be determined via a noninvasive approach, could serve as a routine test to asses the potential impact of the coronary artery disease on the left ventricular function and therefore estimate the probability of underlying severe coronary artery lesions in patients with decrease left ventricular function.

\section{Conclusions}

High Calcium score is positively correlated with LVEF reduction, and a high value for calcium score indicates an

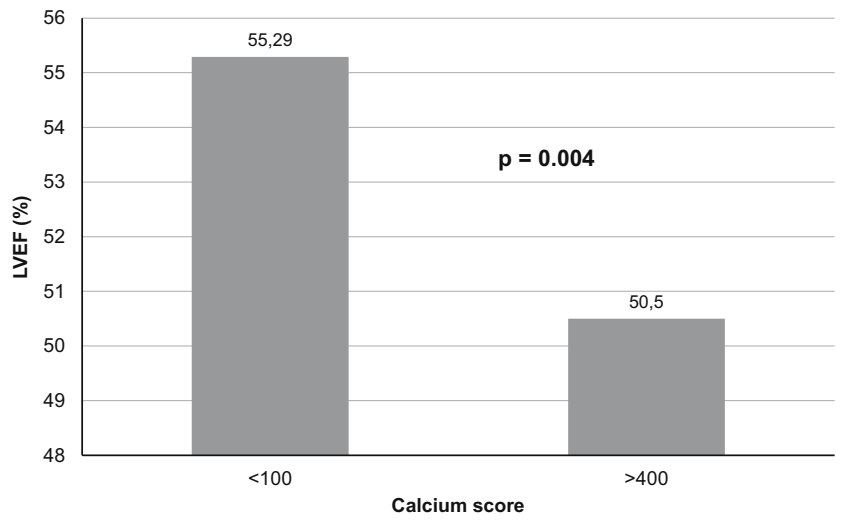

Fig. 3. Calcium score and LVEF

increased probability of reduced left ventricular ejection fraction.

\section{Acknowledgement}

This paper is partly supported by the Sectorial Operation al Programme Human Resources Development (SOP HRD), financed from the European Social Fund and by the Romanian Government under the contract number POSDRU 80641.

\section{References}

1. Meijboom WB, Meijs MF, Schuijf JD, et al. Diagnostic accuracy of 64-slice computed tomography coronary angiography: a prospective, multicenter, multivendor study. J Am Coll Cardiol. 2008;52:2135-2144.

2. Bluemke DA, Achenbach S, Budoff $M$, et al. Noninvasive coronary artery imaging: magnetic resonance angiography and multidetector computed tomography angiography: A Scientific Statement From the American Heart Association Committee on Cardiovascular Imaging and Intervention of the Council on Cardiovascular Radiology and Intervention, and the Councils on Clinical Cardiology and Cardiovascular Disease in the Young. Circulation. 2008;118:586-606.

3. Greenland P, Bonow RO, Brundage BH, et al. ACCF/AHA 2007 Clinical Expert Consensus Document on Coronary Artery Calcium Scoring By Computed Tomography in Global Cardiovascular Risk Assessment and in Evaluation of Patients With Chest Pain. Journal of the American College of Cardiology. 2007;49(3):378-402.

4. Benedek T, Gyongyosi M, Benedek I. Multislice Computed Tomographic Coronary Angiography for Quantitative Assessment of Culprit Lesions in Acute Coronary Syndromes. Canadian Journal of Cardiology. 2013;29(3):364-371.

5. Raggi P, Khan A, Arepali C, Stillman AE. Coronary artery calcium scoring in the age of CT angiography: what is its role? Curr Atheroscler Rep. 2008; 10(5):438-43.

6. Mark DB, Berman DS, Budoff MJ. ACCF/ACR/AHA/NASCI/SAIP/SCAI/ SCCT 2010 Expert Consensus Document on Coronary Computed Tomographic Angiography: A report of the American College of Cardiology Foundation Task Force on Expert Consensus Documents. J Am Coll Cardiol. 2010;55;2263-2699.

7. Schroeder S, Achenbach S, Bengel F, et al. Cardiac computed tomography: indications, applications, limitations, and training requirements. European Heart Journal. 2008;29:531-556.

8. Benedek T, Bucur O, Pascanu I, Benedek I. Analysis of coronary plaque morphology by 64-multislice computed tomography coronary angiography and calcium scoring in patients with type 2 diabetes mellitus. Acta endocrinologica. 2011;7(1):59-68.

9. Colletti M, Dustin L, Wong N, et al. Does Coronary Calcium Score Predict Future Cardiac Function? Association of Subclinical Atherosclerosis with Left Ventricular Systolic and Diastolic Dysfunction at MR Imaging in an Elderly Cohort. Radiology. 2010;257(1):64-70.

10. Edvardsen T, Detrano R, Rosen BD, et al. Coronary Artery Atherosclerosis Is Related to Reduced Regional Left Ventricular Function in Individuals Without History of Clinical Cardiovascular Disease. Arterioscler Thromb Vasc Biol. 2006;26:206-211. 